\title{
Growth Kinetics and Morphology of Colonies of the Filamentous Form of Candida albicans
}

\author{
By NEIL A. R. GOW* AND GRAHAM W. GOODAY \\ Department of Microbiology, University of Aberdeen, Marischal College, \\ Aberdeen AB9 IAS, U.K.
}

(Received 14 December 1981; revised 9 February 1982)

The growth and development of mycelia of the dimorphic fungus Candida albicans in serumcontaining medium is described. Initially, colonies are undifferentiated (all hyphae in the mycelium having approximately the same diameter, extension rate, apical and intercalary compartment lengths) whereas older mycelia differentiate at the colony margin to produce leading hyphae that are wider, faster growing and have longer apical and intercalary compartment lengths than the branches they subtend. Early colony development exhibits unusual features: germ tube extension is linear (not exponential as in other fungi) and there is a prolonged delay between septation and the onset of branch formation. The subsequent patterns of growth and branching are similar in all other respects to those of other mycelial moulds. Mycelia have septa that delimit single nuclei within compartments. The septa do not prevent cytoplasmic flow and consequently allow the peripheral growth zone to span several compartments. From these results we conclude that filamentous growth of $C$. albicans in this medium is best described as truly mycelial.

\section{INTRODUCTION}

Candida albicans is the aetiological agent of thrush and other superficial and systemic diseases of man. The organism can exist as a yeast or in a filamentous form and is readily cultured in vitro in either growth phase. The pattern of budding of the yeast form is closely analogous to that of Saccharomyces cerevisiae (Shannon \& Rothman, 1971; Bedell et al., 1980). The filamentous growth form, which is invasive and therefore thought to be responsible for the disease condition (Blythe, 1959), has been less well characterized and has been variously described as 'pseudomycelial' with 'pseudohyphae', or truly mycelial. Colony growth and development of mycelial fungi is a co-ordinated process that involves replication of a basic growth unit (Trinci, 1974). The cellular processes of growth, nuclear division, septation and branching are regulated and form a cycle of events, termed the duplication cycle (Trinci, 1979), analogous to the cell cycle in unicells. In mycelial fungi hyphae extend only at the hyphal tips (Gooday, 1971; Trinci \& Saunders, 1977). Apical growth is supported by a volume of sub-apical cytoplasm (the peripheral growth zone) which may extend for considerable distances behind the hyphal apex and span septate regions if the cross-walls have pores (Trinci, 1971 a). Regular branching produces an exponentially increasing number of tips so that while individual hyphae extend linearly, growth of the whole mycelium is exponential (Trinci, 1969). Logarithmic relationships have been found between branch orders and the numbers and lengths of branches in Thamnidium elegans (Gull, 1975 ) and the actinomycete Streptomyces viridochromogenes (E. Allan \& J. I. Prosser, personal communication) implying underlying control mechanisms over the pattern of branch production.

This paper describes the growth and development of colonies of the filamentous form of $C$. albicans and discusses the status of this growth form in relation to that of other filamentous fungi. 


\section{METHODS}

Organism and culture media. Candida albicans (Robin) Berkhout strain 3153 was from the London Mycological Reference Laboratory. Newborn calf serum (Gibco Bio-cult, Glasgow) was used in solid or liquid media at a concentration of $20 \%(\mathrm{v} / \mathrm{v})$. For solid media, serum at $50^{\circ} \mathrm{C}$ was mixed with molten agar at the same temperature and dispensed into Petri dishes at $15 \mathrm{ml}$ per plate.

Inoculation and slide culture techniques. Yeast cells were grown for $16 \mathrm{~h}$ at $37^{\circ} \mathrm{C}$ on Sabouraud dextrose agar slopes. Cells were washed from the slopes with sterile distilled water and the density of the resulting suspension determined by counting in an Improved Neubauer haemocytometer. An appropriate volume of cells from a serial dilution was spread on a plate to give approximately $100-200$ cells per plate. A slab of this serum agar approximately $4 \times 2 \mathrm{~cm}$ was cut out and placed on a microscope slide and overlaid with autoclaved polypropylene membrane. The slide was placed on a Reichert heated microscope stage at $37^{\circ} \mathrm{C}$ for observation. Water loss from the agar was prevented by placing wet filter paper strips around the margin of the agar block and keeping these moist. Using this method no visible shrinkage of the agar was detectable even after $15 \mathrm{~h}$. Colonies were photographed at 10 or 20 min intervals using bright-field microscopy.

Measurements from photographs. Measurements of total lengths of mycelia and numbers of branches were made from projections of negatives on to a white board at final magnifications of about $\times 400$ for early colonies and $\times 100$ for late colonies. Extension rates were calculated from these lengths over known time intervals. Diameters of hyphae were calculated from similar projections at higher magnifications and are given as the average of the outer and inner phase haloes across the hypha. Apical and sub-apical compartment lengths were measured directly from specimens using an eye piece graticule calibrated against a micrometer slide. Measurements on photographs were calibrated from projections of this micrometer slide.

Colony radial growth rate. The diameter of colonies on $20 \%$ serum agar (with and without polypropylene membrane overlays) was measured across perpendicular meridia at daily intervals.

Determination of the hyphal growth unit and the mean tip extension rate. The hyphal growth unit $(G)$ (Trinci, 1974) was calculated from the equation:

$$
G=\text { Total length of mycelium }(\mu \mathrm{m}) / \text { No. of tips }
$$

The mean rate of tip extension $\left(E ; \mu \mathrm{m}\right.$ per tip $\left.\mathrm{h}^{-1}\right)$ (Trinci, 1974) was measured for colonies with between 10 and 20 tips.

$$
E_{\text {measured }}=2\left(H_{\mathrm{t}}-H_{0}\right) /\left(B_{0}+B_{\mathrm{t}}\right)
$$

where $H_{0}=$ total hyphal length at zero time, $H_{\mathrm{t}}=$ total length $1 \mathrm{~h}$ later, $B_{0}=$ number of tips at zero time, and $B_{\mathrm{t}}$ $=$ number of tips $1 \mathrm{~h}$ later.

$E$ was also calculated from the equation (Steele \& Trinci 1975)

$$
E=G \alpha
$$

where $G$ is the hyphal growth unit and $\alpha$ is the specific growth rate of the organism, which was determined from the doubling time $\left(t_{\mathrm{d}}, \mathrm{h}\right)$ of the total mycelial length:

$$
\alpha=\ln 2 / t_{\mathrm{d}}
$$

Calculation of the peripheral growth zone. The peripheral growth zone $(w)$ was calculated from the extension rate of a colony or hypha $\left(K_{\mathrm{r}}\right)$ and the specific growth rate $(\alpha)$ as:

$$
w=K_{\mathrm{r}} / \alpha
$$

All measurements are given as mean values \pm standard deviations of the stated number of replicates.

Analysis of branching. Analysis of branching patterns of mycelia was carried out using the method of Leopold (1971). In this system, hyphae are classified by orders according to the branches they subtend. First order hyphae subtend no branches, second order branches subtend only first order branches and third order hyphae subtend second and first order branches. The length of a branch of a particular order is taken as the length from the apex of that branch to the junction of the branch of the next highest order. The antilogs of the slopes of the plots of the logarithm of the number of branches against the branch order, and the logarithm of average branch length against the branch order (called the branch ratio and length ratio, respectively) were calculated for mycelia with at least one third order hypha. Mycelia with hyphae with higher order numbers than three were too complex to analyse readily.

Staining of septa and nuclei. Mycelia were filtered from media or washed from membranes and fixed in $70 \%(\mathrm{v} / \mathrm{v})$ ethanol for $30 \mathrm{~min}$. The ethanol was then removed and the material stained with 4',6-diaminido-2-phenylindole (DAPI, Sigma; $2 \mu \mathrm{g} \mathrm{ml}^{-1}$ ) in $200 \mathrm{~mm}$-sodium phosphate buffer, pH $7 \cdot 5$, containing calcofluor white $(0 \cdot 025 \mathrm{mg}$ $\mathrm{ml}^{-1}$; American Cyanamid Co., Chicago). Samples were examined under epifluorescence with a Reichert Zetopan microscope with 400 exciter and 400 barrier filters. Septa and nuclei fluoresced bright blue under these conditions. 


\section{RESULTS}

Figure 1 shows the growth of a germ tube that emerged from a yeast cell inoculated on to serum agar. The extension rate was constant and rates were very reproducible between different germ tubes (Table 1). There was no observable change in size of the yeast itself during this process.

The kinetics of the increase in total hyphal length and the numbers of tips are shown in Fig. 2, and the final appearance of this mycelium is illustrated in Fig. 3. Linear growth of the germ tube resulted in an initial hyperbolic relationship for $\log$ (total mycelial length) against time. Up to four germ tubes have been seen emerging from a single mother yeast cell. Initial discontinuous tip production gradually became continuous as the mycelium increased in length; consequently, overall growth of the mycelium became exponential (at the same specific rate as for tip production) though individual branches extended linearly. The hyphal growth unit initially increased then oscillated before attaining a constant average length of 48.1 $\pm 9.4 \mu \mathrm{m}$ (11 different mycelia). The mean tip extension rate for these mycelia was measured [as in equation (2)] to be $18.8 \pm 2.4 \mu \mathrm{m} \mathrm{h}^{-1}$ and calculated from equation (3) as $18.5 \mu \mathrm{m} \mathrm{h}^{-1}$. Hyphae did not exhibit negative autotropism and were seen to cross one another frequently, resulting in an uneven distribution of the mycelium over the substrate (Fig. 3).

The pattern of septation in the first germ tube of a developing mycelium is shown in Fig. 4. Septa (S1-S5) were laid down at approximately hourly intervals and divided the germ tube into

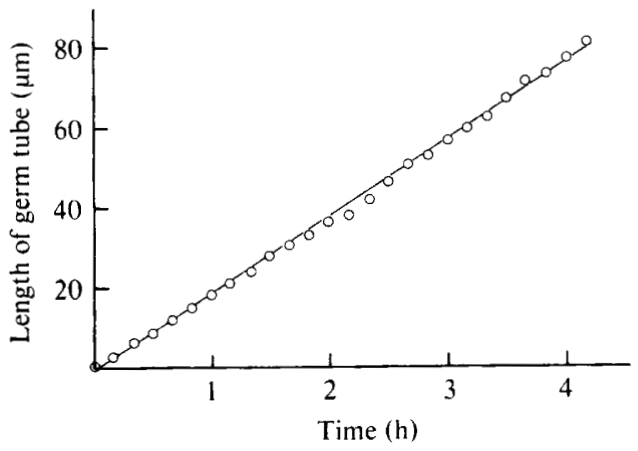

Fig. 1. Growth of a germ tube of C. albicans from a yeast cell on $20 \%$ serum agar at $37^{\circ} \mathrm{C}$.

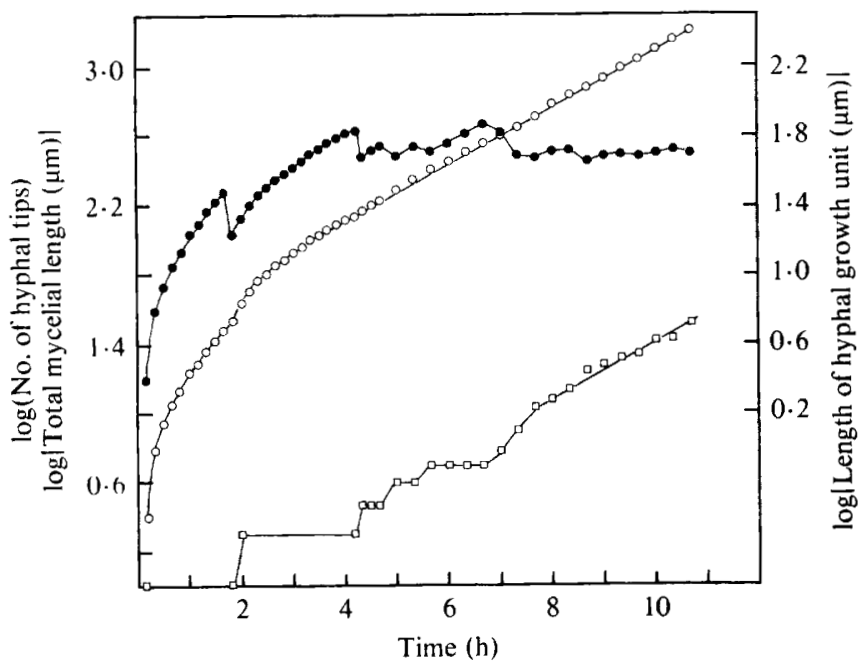

Fig. 2. Number of tips ( $\square$ ), total hyphal length $(O)$ and length of the hyphal growth unit $(O)$ in a developing mycelium of $C$. albicans on $20 \%$ serum agar at $37^{\circ} \mathrm{C}$. 


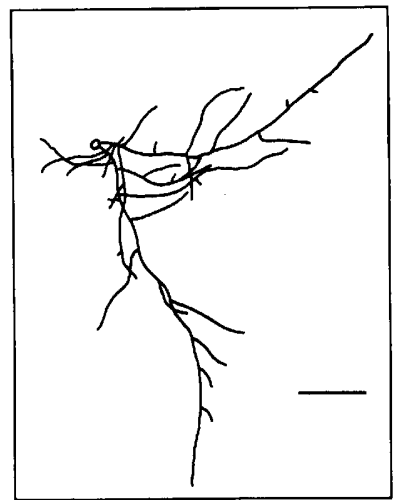

Fig. 3. Appearance of mycelium described in Fig. $210.67 \mathrm{~h}$ after germ tube formation. The scale bar represents $50 \mu \mathrm{m}$.

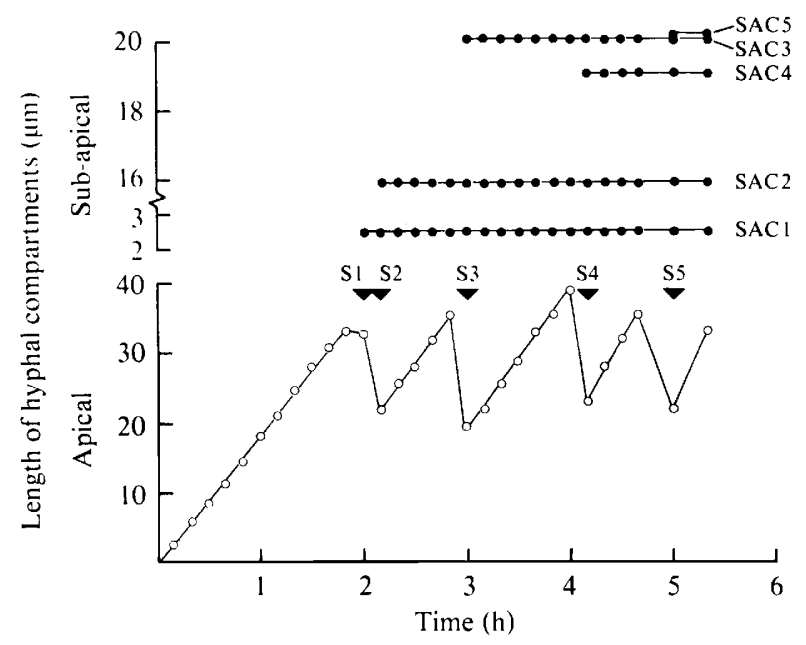

Fig. 4. Pattern of septation in a germ tube of $C$. albicans. Septa (S1-S5) were first seen at the times indicated by the arrows and divided the germ tube into apical and sub-apical (intercalary) compartments (SAC1-SAC5).

apical and sup-apical (intercalary) compartments (SAC1-SAC5). Septation occurred without interruption in extension rate. The first septum S1 delimited the original yeast cell from the growing hypha at a position close to its point of origin. The first sub-apical compartment (SAC1) was therefore considerably shorter than subsequent compartments (SAC2-SAC5), which were approximately the same length (cf. Table 1). Once formed, intercalary compartments did not further extend. In this germ tube the first branch initial was formed just behind the septum $4.33 \mathrm{~h}$ after germ tube formation. The delay between septation and the onset of branch production was a reproducible feature.

The branch and length ratios of a mycelium with third order hyphae were calculated from the slopes of the lines in Fig. 5. The average values for the branch and length ratios were $3.86 \pm 1.51$ and $2.38 \pm 0.67$, respectively, for seven separate mycelia. This indicates that the branches of any order will be 3.86 times as plentiful and 2.38 times as long as the branches in the next lowest order.

Table 1 shows the characteristics of different branch types in early (undifferentiated) and older (differentiated) colonies. In undifferentiated colonies the extension rates, hyphal diame- 


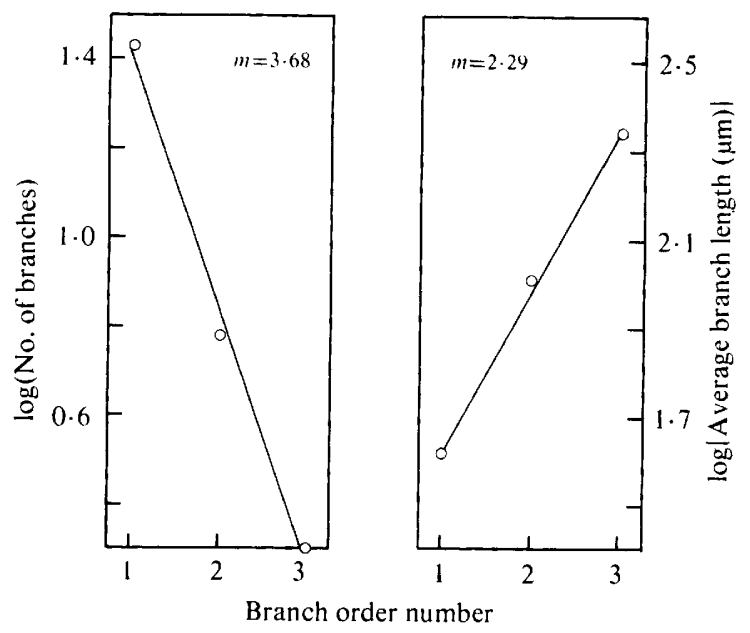

Fig. 5. Plots of the logarithm of the number of branches and of the length of branches of various orders in a third order mycelium of $C$. albicans. The values of the antilog of the slopes of the lines $(m)$, indicating the branch and length ratios, respectively, are given.

Table 1. Morphology and extension rates of various branch types in early (undifferentiated) and mature (differentiated) mycelia of C. albicans

All values are mean values $(\mu \mathrm{m}) \pm$ S.D. for 20 hyphae, except for extension rates $\left(\mu \mathrm{m} \mathrm{h} \mathrm{h}^{-1}\right)$, which are mean values for 6-20 hyphae.

Diameter of hyphae

Apical compartment length

1 st intercalary compartment length

2nd intercalary compartment length

3rd intercalary compartment length

Extension rate

$\begin{array}{ccc}\begin{array}{c}\text { Undifferentiated mycelia } \\ (12 \text { h colonies })\end{array} \\ \begin{array}{c}\text { Germ } \\ \text { tubes }\end{array} & \begin{array}{c}\text { Primary } \\ \text { branches }\end{array} & \begin{array}{c}\text { Secondary } \\ \text { branches }\end{array} \\ 2 \cdot 6 \pm 0.2 & 2 \cdot 4 \pm 0 \cdot 2 & 2 \cdot 3 \pm 0 \cdot 2 \\ 35.7 \pm 7 \cdot 1 & 30.8 \pm 5 \cdot 5 & \text { ND } \\ 23 \cdot 1 \pm 3 \cdot 2 & 21.3 \pm 3 \cdot 8 & \text { ND } \\ 22.5 \pm 5.6 & 19.3 \pm 2 \cdot 5 & \text { ND } \\ 22 \cdot 1 \pm 2.5 & \text { ND } & \text { ND } \\ 19.3 \pm 2.9 & 18.9 \pm 2 \cdot 2 & \text { ND }\end{array}$

ND, Not determined.
Differentiated mycelia (3-5 day colonies)

$\overbrace{\text { Leading Primary Secondary }}$

hyphae branches branches

$3.4 \pm 0.3 \quad 2 \cdot 5 \pm 0.2 \quad 2 \cdot 3 \pm 0.2$

$51.9 \pm 9.844 .7 \pm 10.4 \quad$ ND

$33.5 \pm 2.0 \quad 27.8 \pm 4 \cdot 7 \quad$ ND

$33.9 \pm 2.028 .9 \pm 4.6 \quad$ ND

$31.8 \pm 6.427 .9 \pm 3 \cdot 1 \quad$ ND

$45.8 \pm 5.6 \quad 30.4 \pm 8.5 \quad 21.9 \pm 6.0$

ters and lengths of apical and intercalary compartments of germ tubes and primary branches (branches that emerge from the germ tube) were approximately the same. Older mycelia became differentiated at the colony margin and had 'leading hyphae', which determined the radial growth rate of the colony and which had faster extension rates, wider diameters and longer apical and intercalary compartments than the primary and secondary branches they subtended. (Secondary branches are those that emerge from primary branches.) The angles between branches and parent hyphae were random and did not tend to $90^{\circ}$ as in undifferentiated mycelia of Neurospora crassa (Steele \& Trinci, 1975).

Colonies older than $15 \mathrm{~h}$ increasingly formed lateral buds on hyphae behind the apical region where no buds were seen (Fig. 6) and became fluffy in appearance due to the production of aerial hyphae. Mature colonies were grown without interruption to the colony radial growth for up to two weeks, after which disc inocula were removed and placed on fresh agar. Mycelia of $C$. albicans can be maintained indefinitely by this method.

The peripheral growth zones of hyphae of undifferentiated mycelia and leading hyphae of differentiated mycelia were calculated from equations (4) and (5) as 48 and $119 \mu \mathrm{m}$ respectively. 


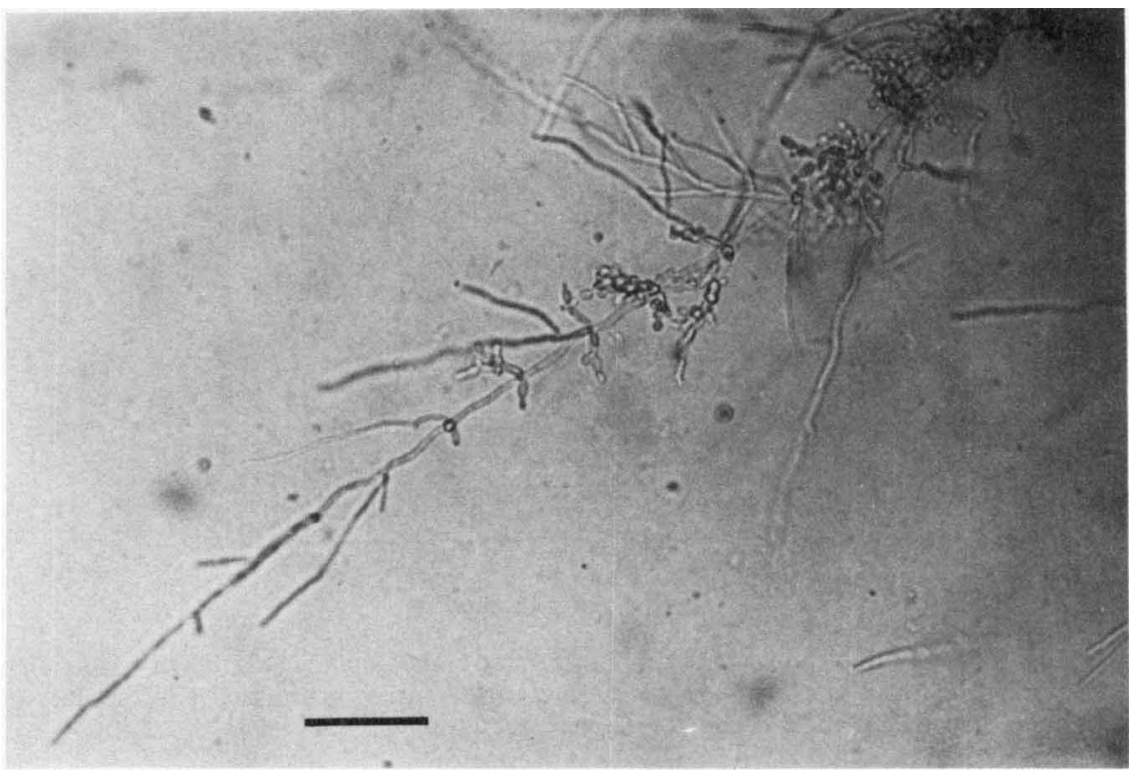

Fig. 6. Margin of a $5 \mathrm{~d}$ colony of $C$. albicans showing extensive lateral bud formation behind the apical region. The scale bar represents $50 \mu \mathrm{m}$.
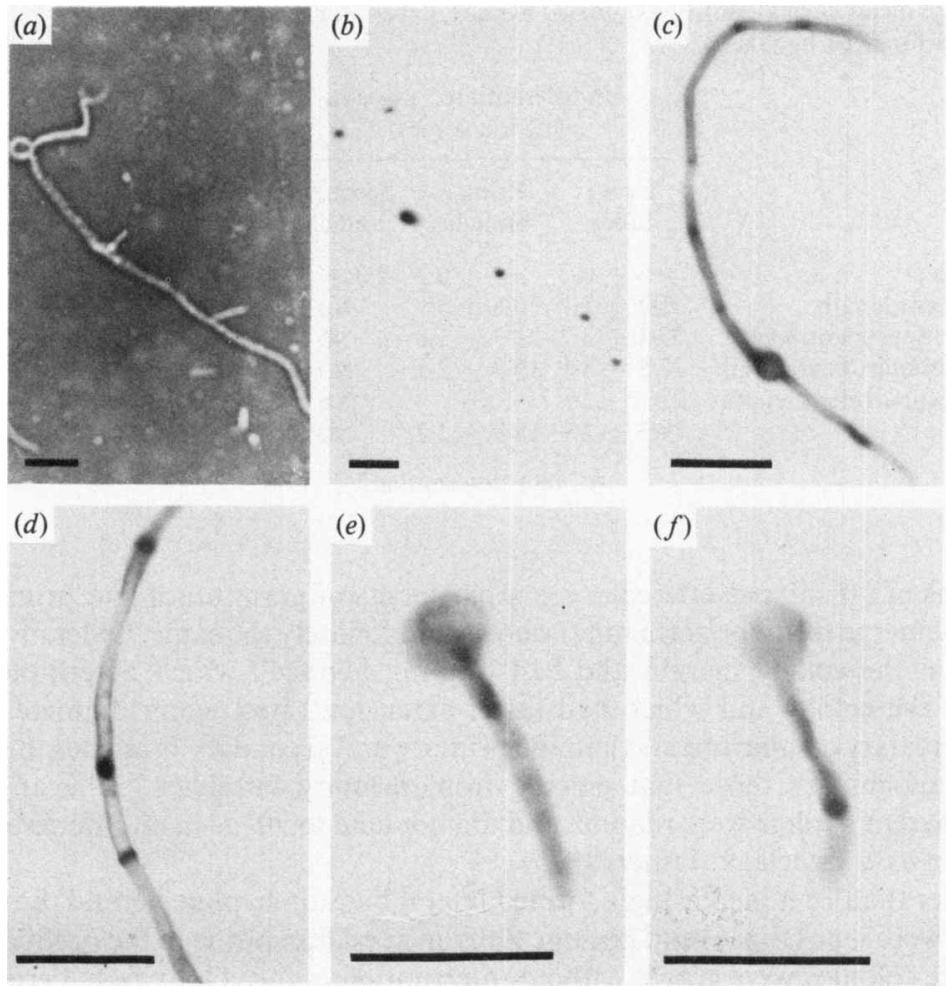

Fig. 7. Mycelia of $C$. albicans under bright-field $(a)$ and epifluorescence $(b-e)$. Mycelia are stained with DAPI $(b)$ or DAPI plus Calcofluor $(c-e)$. Single nuclei are compartmented by septa in germ tubes $(c)$ and hyphae $(d)$. In early germlings $(e, f)$ nuclei migrate as elongated structures $(e)$ before dividing $(f)$. Photographs $(b-e)$ are negatives from original positives on Ektachrome 200. The scale bars represent $20 \mu \mathrm{m}$. 
The small size of the hyphae has made it impossible to determine the peripheral growth zone experimentally by cutting of the mycelium (Trinci, 1971 b) and the linear outgrowth of germ tubes precluded estimations of the peripheral growth zone by measurements of germ tubes when exponential extension (characteristic of the germination process of several other fungi) started to decelerate to a linear extension rate. The tendency of mycelia of $C$. albicans to form large multinucleated clumps when in shake flasks prevented measurement of the specific growth rate in liquid culture as in other studies (Trinci, 1969, 1970). The specific growth rate $\alpha$ in equations (4) and (5) was determined instead from the slopes of lines for total mycelial lengths against time (cf. Fig. 2) as $0.394 \pm 0.047 \mathrm{~h}^{-1}$ (11 different mycelia).

In mycelia stained with calcofluor and DAPI, nuclei were seen to be delimited in compartments, one nucleus per compartment (Fig. 7). In no instance other than in apical compartments or compartments with newly initiated branches was more than one nucleus found within a single compartment.

\section{DISCUSSION}

Colony formation and development of the mycelial form of $C$. albicans closely resembles that of other filamentous fungi such as Neurospora crassa (Steele \& Trinci, 1975) but exhibits some unusual features. Mycelial growth is similar to that of other fungi in that the total mycelial length and the rate of tip (branch) production becomes exponential with the same specific growth rate (Trinci, 1974). Also, as in many other fungi, mycelia differentiate at the edge of mature colonies to produce wider, faster 'leading hyphae', which enable rapid substrate-colonization. Early colony formation differs from that of other fungi in that germ tube extension is always linear and never exponential, and that branch formation does not occur directly after septation, but is delayed until several further septa have been laid down behind the hyphal apex. These differences are difficult to explain in terms of present models of hyphal growth (Prosser \& Trinci, 1979), which predict that exponential extension would occur in any hyphal system with vesicular transport and apical growth. It is known that hyphae of $C$. albicans incorporate wall precursors at their apices (Braun \& Calderone, 1978). Linear growth would be explicable if either the physiology of hyphal growth was different or, alternatively, if growth was constrained in these hyphae. Diameters of hyphae in Table 1 are larger than those measured in thin section (Gow et al., 1980) but agree closely with measurements using Nomarski interference microscopy (unpublished data). These narrow hyphae may be restrictive to vesicle transport. Alternatively, narrow hyphae with a high surface area to volume ratio may be able to absorb sufficient nutrients from the environment to immediately saturate the biosynthetic capacity of the apex and achieve maximum extension rates more quickly than wider hyphae. The lack of exponential growth here may also be related to the difference between the formation of the germ tube from a yeast cell of C. albicans and spore germination of a filamentous fungus in that here there is no requirement for a general activation of metabolic processes and no observable swelling. The yeast cell can presumably immediately contribute to growth of the germ tube. A preliminary estimate of the average yeast cell volume of $93 \mu \mathrm{m}^{3}$ is about half that estimated for the volume of the peripheral growth zone of the germ tube $\left(180 \mu \mathrm{m}^{3}\right)$. Thus the yeast cell cytoplasm cannot be equated totally with a hyphal peripheral growth zone in its ability to support the observed immediate linear growth of the germ tube. Linear growth (as measured by increase in total dry mass) occurs over the cell cycles of the unicellular fungi Saccharomyces cerevisiae (Mitchison, 1958; Scopes \& Williamson, 1964) and Schizosaccharomyces pombe (Mitchison, 1957).

In the duplication cycle of moulds (Trinci, 1979), septation is usually followed by branch formation behind a septal plate. This is thought to be due to the diversion of vesicles thus isolated from the hyphal apex into branch formation and it results in a doubling in the rate of biomass production. The delay seen here between branch point formation and branch emergence may reflect differences in the control of branching or in the internal distribution of vesicles, which again may be related to the narrowness of the hyphae.

The filamentous form of $C$. albicans is stable and can be maintained by subculturing on serum agar. The noticeable increase in the proportion of yeast cells to filamentous entities after several hours in liquid culture does not occur because of a gradual reversion to the unicellular state, but 
rather because of the production of lateral buds, which are formed behind the tips of marginal hyphae of developing colonies.

The peripheral growth zone is longer than the apical compartment lengths of both undifferentiated and differentiated mycelia. This provides indirect evidence for the presence of a septal pore which has been shown to be present and is of a size too small to allow nuclear migration while maintaining cytoplasmic continuity (Gow et al., 1980). The ordered distribution of nuclei found within mycelia is thus maintained by the septal architecture, which allows the peripheral growth zone to span septal regions and thus enhance the maximum possible extension rate.

As the hyphae consist of uninucleated compartments delimited by septa, they might be likened to a chain of expanded cells joined end to end (pseudomycelium). However, the cytoplasm between adjacent cells is contiguous and there is no evidence of intercalary extension characteristic of pseudomyceliation. This growth form is best described as being truly mycelial.

We thank Dr J. I. Prosser for helpful discussions.

\section{REFERENCES}

Bedell, G. W., Werth, A. \& Soll, D. R. (1980). The regulation of nuclear migration and division during synchronous bud formation in released stationary phase cultures of the yeast Candida albicans. Experimental Cell Research 127, 103-113.

BlyTHE, W. (1959). Host/parasite relationships in experimental moniliasis. I. Candida albicans. Mycopathologia 10, 269-282.

Braun, P. C. \& Calderone, R. A. (1978). Chitin synthesis in Candida albicans: comparison of yeast and hyphal forms. Journal of Bacteriology 135, 14721477.

GoODAY, G. W. (1971). An autoradiographic study of hyphal growth of some fungi. Journal of General Microbiology 67, 125-133.

Gow, N. A. R., Gooday, G. W., Newsam, R. J. \& GULL, K. (1980). Ultrastructure of the septum in Candida albicans. Current Microbiology 4, 357-359.

GuLL, K. (1975). Mycelial branch patterns of Thamnidium elegans. Transactions of the British Mycological Society 64, 321-324.

LEOPOLD, L. B. (1971). Trees and streams: the efficiency of branching patterns. Journal of Theoretical Biology 31, 339-354.

MrTchison, J. M. (1957). The growth of single cells. I. Schizosaccharomyces pombe. Experimental Cell Research 13, 244-262.

Mitchison, J. M. (1958). The growth of single cells. II. Saccharomyces cerevisiae. Experimental Cell Research 15, 214-221.

Prosser, J. I. \& Trinci, A. P. J. (1979). A model for hyphal growth and branching. Journal of General Microbiology 111, 153-164.

Scopes, A. W. \& Williamson, D. H. (1964). The growth and oxygen uptake of synchronously dividing cultures of Saccharomyces cerevisiae. Experimental Cell Research 35, 361-371.

Shannon, J. L. \& Rothman, A. L. (1971). Transverse septum formation in budding cells of the yeast-like fungus Candida albicans. Journal of Bacteriology 106, 1026-1028.

Steele, G. C. \& Trinci, A. P. J. (1975). Morphology and growth kinetics of hyphae of differentiated and undifferentiated mycelia of Neurospora crassa. Journal of General Microbiology 91, 362-368.

TrinCI, A. P. J. (1969). A kinetic study of the growth of Aspergillus nidulans and other fungi. Journal of General Microbiology 57, 11-24.

TrINCI, A. P. J. (1970). Kinetics of the growth of mycelial pellets of Aspergillus nidulans. Archiv für Mikrobiologie 73, 353-367.

TRINCI, A. P. J. (1971a). Exponential growth of the germ tubes of fungal spores. Journal of General Microbiology 67, 345-348.

TRINCI, A. P. J. (1971 b). Influence of the peripheral growth zone on the growth of Aspergillus nidulans and other fungi. Journal of General Microbiology 67, 325-344.

TrINCI, A. P. J. (1974). A study of the kinetics of hyphal extension and branch initiation of fungal mycelia. Journal of General Microbiology 81, 225236.

TrincI, A. P. J. (1979). The duplication cycle and branching in fungi. In Fungal Walls and Hyphal Growth, pp. 319-358. Edited by J. H. Burnett \& A. P. J. Trinci. Cambridge: Cambridge University Press.

Trinci, A. P. J. \& SAunders, P. T. (1977). Tip growth of fungal hyphae. Journal of General Microbiology 103, 243-248. 